\title{
The binary pulsar PSR J1811-1736: evidence of a low amplitude supernova kick
}

\author{
A. Corongiu ${ }^{1,2,3}$, M. Kramer ${ }^{3}$, B. W. Stappers ${ }^{4}$, A. G. Lyne ${ }^{3}$, A. Jessner ${ }^{5}$, A. Possenti ${ }^{2}$, \\ N. D'Amico ${ }^{1,2}$, and O. Löhmer ${ }^{5}$ \\ 1 Università degli Studi di Cagliari, Dip. di Fisica, SP Monserrato-Sestu km 0,700, 09042 Monserrato, Italy \\ e-mail: corongiu@ca.astro.it \\ 2 INAF - Osservatorio Astronomico di Cagliari. Loc. Poggio dei Pini, Strada 54, 09012 Capoterra, Italy \\ 3 University of Manchester, Jodrell Bank Observatory, Jodrell Bank, Macclesfield, Cheshire SK11 9DL, UK \\ 4 Stichting ASTRON, Postbus 2, 7990 AA Dwingeloo, The Netherlands \\ 5 MPI für Radioastronomie, Auf dem Hügel 69, 53121 Bonn, Germany
}

Received 20 October 2005 / Accepted 1 November 2006

\section{ABSTRACT}

\begin{abstract}
Aims. The binary pulsar PSRJ1811-1736 has been identified, since its discovery, as a member of a double neutron star system. Observations of such binary pulsars allow the measurement of general relativistic effects, which in turn lead to information about the orbiting objects, to their binary evolution and, in a few cases, to tests of theories of gravity.

Methods. Regular timing observations have since January 1999 been carried out with three of the largest European radio telescopes involved in pulsar research. Pulse times of arrival were determined by convolving the observed profiles with standard templates, and were fitted to a model that takes into account general relativistic effects in binary systems. The prospects of continued observations were studied with simulated timing data. Pulse scattering times were measured using dedicated observations at $1.4 \mathrm{GHz}$ and at $3.1 \mathrm{GHz}$, and the corresponding spectral index has also been determined. The possibility of detecting the yet unseen companion as a radio pulsar was investigated as a function of pulse period, observing frequency and flux density of the source. A study of the natal kick received by the younger neutron star at birth was performed considering the total energy and total angular momentum for a two body system.

Results. We present an up to date and improved timing solution for the binary pulsar PSR J1811-1736. One post-Keplerian parameter, the relativistic periastron advance, is measured and leads to the determination of the total mass of this binary system. Measured and derived parameters strongly support the double neutron star scenario for this system. The pulse profile at $1.4 \mathrm{GHz}$ is heavily broadened by interstellar scattering, limiting the timing precision achievable at this frequency. We show that a better precision can be obtained with observations at higher frequencies. This would allow one to measure a second post-Keplerian parameter within a few years. We find that interstellar scattering is unlikely to be the reason for the continued failure to detect radio pulsations from the companion of PSR J1811-1736. The probability distribution that we derive for the amplitude of the kick imparted on the companion neutron star at its birth indicates that the kick has been of low amplitude.
\end{abstract}

Key words. pulsar: general - pulsars: individual: PSR J1811-1736

\section{Introduction}

The pulsar J1811-1736, discovered during observations of the Parkes Multibeam Pulsar Survey (Manchester et al. 2001), has a spin period of $104 \mathrm{~ms}$ and is member of a 18.8-d, highly eccentric binary system (Lyne et al. 2000) with an as yet undetected companion (Mignani 2000). The characteristic age and the estimated surface magnetic field strength indicate that the pulsar is mildly recycled. In such a system, it is expected that the observed pulsar is born first in a supernovae (SN) explosion before it undergoes mass accretion from a high-mass non-degenerate binary companion. Parameters which have been measured and derived from the best-fit timing solution indicate that the companion is quite massive. All these elements suggest that the companion is also a neutron star (e.g. Bhattacharya \& van den Heuvel 1991).

The conclusion that PSR J1811-1736 is a member of the small sample of known double neutron star (DNS) systems was already reached by Lyne et al. (2000). Their conclusion was supported by a constraint on the total system mass, assuming that the observed advance of periastron was totally due to relativistic effects. However, the rather long period of the pulsar, combined with the effects of interstellar scattering, resulting in significant broadening of the pulse profile at $1.4 \mathrm{GHz}$, as well as the short data span available to Lyne et al. (2000), limited the timing precision and hence the accuracy of the total mass measurement.

Among all DNS systems, PSR J1811-1736 has by far the longest spin period, the longest orbital period and the highest eccentricity. This may suggest that the evolution of this DNS has been different, at least in part, from all other DNS systems. On the other hand, PSR J1811-1736 well fits the spin period versus eccentricity relation for DNS systems (McLaughlin et al. 2005, Faulkner et al. 2005). This relation can be simply explained in terms of the different lengths of time the pulsar underwent accretion, which in turn is related to the mass of the companion star before the SN explosion. Moreover, numerical simulations (Dewi et al. 2005) show that the spin period versus eccentricity relation is recovered assuming that the second born neutron star received a low velocity kick at its birth.

In this paper we report on new timing observations which significantly improve on the previously published results. We present a study of the observed interstellar scattering and 
consider its consequences on the detectability of radio pulses from the companion. Finally, we investigate the likely kick velocity imparted to the second-born neutron star during its birth in the system's second SN. Observations were carried out as part of a coordinated effort using three of the largest steerable radio telescopes in the world for pulsar timing observations, i.e. the 100-m radio telescope at Effelsberg, the 94-m equivalent Westerbork Synthesis Radio Telescope (WSRT) and the 76-m Lovell telescope at Jodrell Bank telescope. This paper is the first in a series detailing results of these efforts in establishing a European Pulsar Timing Array (EPTA).

\section{Observations}

The binary pulsar J1811-1736 is one of the binary pulsars regularly observed by the EPTA. The aims and objectives of this European collaboration include the detection of a cosmological gravitational wave background and the project will be described in detail in a forthcoming publication. Here we summarize the observing systems used while further details can be found in the references below.

\subsection{Effelsberg timing}

We made regular timing observations of PSR J1811-1736 since October 1999 using the 100-m radio telescope of the Max Planck Institut für Radioastronomie in Effelsberg near Bonn. The typical observing rate was of 1 observation every two months. An overall root-mean-square (rms) of $538 \mu \mathrm{s}$ is achieved after applying the final timing model. The data were obtained with a 1.3-1.7 GHz tunable HEMT receiver installed in the primary focus of the telescope. The noise temperature of this system is $25 \mathrm{~K}$, resulting in a system temperature from 30 to $50 \mathrm{~K}$ on cold sky depending on elevation. The antenna gain at these frequencies is $1.5 \mathrm{~K} \mathrm{Jy}^{-1}$.

An intermediate frequency (IF) centred on $150 \mathrm{MHz}$ for left-hand (LHC) and right-hand (RHC) circularly polarised signals was obtained after down-conversion from a central RF frequency of usually $1410 \mathrm{MHz}$. The signals received from the telescope were acquired and processed with the Effelsberg-Berkeley Pulsar Processor (EBPP) which removes the dispersive effects of the interstellar medium on-line using "coherent de-dispersion" (Hankins \& Rickett 1975). Before entering the EBPP, the two LHC and RHC signals of the IF are converted to an internal IF of $440 \mathrm{MHz}$. A maximum bandwidth of $2 \times 32 \times 0.7 \mathrm{MHz}=2 \times$ $32 \mathrm{MHz}$ was available for the chosen observing frequency and $\mathrm{DM}$ of the pulsar. It was split into four portions for each of the two circular polarisations, which were mixed down to baseband. Each portion was then sub-divided into eight narrow channels via a set of digital filters (Backer et al. 1997). The outputs of each channel were fed into de-disperser boards for coherent online de-dispersion. In total 64 output signals were detected and integrated in phase with the predicted topocentric pulse period.

A pulse time-of-arrival (TOA) was calculated for each average profile obtained during a 5-10 min observation. During this process, the observed time-stamped profile was compared to a synthetic template, which was constructed out of 5 Gaussian components fitted to a high signal-to-noise standard profile (see Kramer et al. 1998; 1999). This template matching was done by a least-squares fitting of the Fourier-transformed data (Taylor 1992). Using the measured time delay between the actual profile and the template, the accurate time stamp of the data provided by a local H-MASER and corrected off-line to UTC(NIST) using recorded information from the satellites of the Global
Positioning System (GPS), the final TOA was obtained. The uncertainty of each TOA was estimated using a method described by Downs \& Reichley (1983) and Lange (1999).

\subsection{Jodrell Bank timing}

Observations of PSR J1811-1736 were made regularly using the 76-m Lovell telescope at Jodrell Bank since its discovery in 1997 (Lyne et al. 2000). The typical observing rate was of about 2 observations each week, with an overall RMS of $1300 \mu$ s after applying the final timing model. A cryogenic receiver at $1404 \mathrm{MHz}$ was used, and both LHC and RHC signals were observed using a $2 \times 32 \times 1.0-\mathrm{MHz}$ filter bank at $1404 \mathrm{MHz}$. After detection, the signals from the two polarizations were filtered, digitised at appropriate sampling intervals, incoherently de-dispersed in hardware before being folded on-line with the topocentric pulse period and written to disk. Each integration was typically of 1-3 min duration; 6 or 12 such integrations constituted a typical observation. Off-line, the profiles were added in polarisation pairs before being summed to produce a single total-intensity profile. A standard pulse template was fitted to the observed profiles at each frequency to determine the pulse times-of-arrival (TOAs). Details of the observing system and the data reduction scheme can be found elsewhere (e.g. Hobbs et al. 2004).

\subsection{Westerbork timing}

Observations of PSR J1811-1736 were carried out approximately monthly since 1999 August 1st, obtaining an overall timing RMS of $659 \mu$ s after applying the final timing model, at a central frequency of $1380 \mathrm{MHz}$ and a bandwidth of $80 \mathrm{MHz}$. The two linear polarisations from all 14 telescopes were added together in phase by taking account of the relative geometrical and instrumental phase delays between them and then passed to the PuMa pulsar backend (Voûte et al. 2002). The data were obtained with the L-band receiver installed in the primary focus of the telescopes. The noise temperature of this system is $25 \mathrm{~K}$, resulting in a system temperature from 30 to $50 \mathrm{~K}$ on cold sky depending on elevation. The antenna gain at these frequencies is $1.2 \mathrm{~K} \mathrm{Jy}^{-1}$. PuMa was used in its digital filterbank mode whereby the Nyquist sampled signals are Fourier transformed and the polarisations combined to produce total intensity (Stokes I) spectra with a total of 512 channels. These spectra were summed online to give a final sampling time of $409.6 \mu$ s and recorded to hard disk. These spectra were subsequently dedispersed and folded with the topocentric period offline to form integrations of a few minutes. TOAs were calculated for each profile following a scheme similar to that outlined above for Effelsberg data, except a high signal-to-noise standard profile was used instead of Gaussian components. In the future, EPTA timing will employ an identical synthetic template for all telescopes.

\section{Data analysis}

The TOAs, corrected to UTC(NIST) via GPS and weighted by their individual uncertainties determined in the fitting process, were analysed with the TEMPO software package (Taylor \& Weisberg 1989), using the DE405 ephemeris of the Jet Propulsion Laboratory (Standish 1990). TEMPO minimizes the sum of weighted squared timing residuals, i.e. the difference between observed and model TOAs, yielding a set of improved 
Table 1. Data sets' characteristics.

\begin{tabular}{llll}
\hline \hline & Jodrell Bank & Effelsberg & Westerbork \\
\hline N. of ToAs & 348 & 74 & 213 \\
Time span & $50842-53624$ & $51490-53624$ & $51391-53546$ \\
R.M.S. & 1300 & 538 & 659 \\
\hline
\end{tabular}

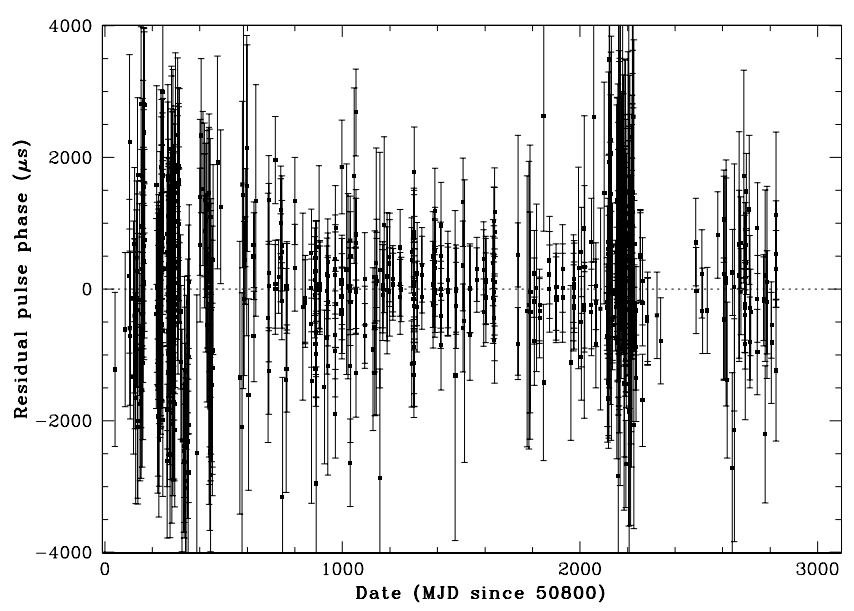

Fig. 1. Timing residuals after jointly applying the final model to all three data sets. Vertical bars represent the ToA's uncertainty.

pulsar parameters and post-fit timing residuals. A summary of the basic characteristics of each dataset is shown in Table 1.

Although the templates used for the three telescope data differed, resulting offsets were absorbed in a global least-squares fit. Remaining uncertainties were smaller than the typical measurement accuracy of the Jodrell Bank timing data of about $9 \mu \mathrm{s}$.

Before all TOAs were combined, preliminary fits were performed on each dataset alone, in order to study possible systematic differences between the datasets. We applied a small quadrature addition and a scaling factor to the uncertainties to obtain the expected value of a reduced $\chi^{2}=1$ for each dataset. The final joint fit to all TOAs resulted in a $\chi^{2}$ value of unity, avoiding the need to add further systematic uncertainties.

Table 2 summarizes all observed timing and some derived parameters. For the observed parameters the quoted errors are twice the nominal TEMPO errors. For the derived parameters, the given uncertainties are computed accordingly.

The joint fit allowed us to determine the spin, positional and Keplerian orbital parameters plus one post-Keplerian parameter with a precision better than the best determination from a single data set alone. However, the high degree of interstellar scattering (Fig. 3) means that further post-Keplerian parameters will be difficult to measure with continued observations at this frequency. We will discuss the future prospects for higher frequency observations in Sect. 5.

\section{The nature of the companion}

In their discovery paper, Lyne et al. (2000) proposed that this system is a member of the small class of DNS binaries. Soon after, Mignani (2000) reported on optical observations of the region surrounding the pulsar position to search for emission from the pulsar companion. They detected no emission coincident with the pulsar position and while not conclusive, the lack of emission is at least consistent with the neutron star hypothesis for the nature of the companion.
Table 2. Timing and derived parameters.

\begin{tabular}{ll}
\hline \hline Timing parameters & Joint data sets \\
\hline RA (J2000, hh:mm:ss) & $18: 11: 55.034(3)$ \\
DECL (J2000, deg:mm:ss) & $-17: 36: 37.7(4)$ \\
Period, $P(\mathrm{~s})$ & $0.1041819547968(4)$ \\
Period derivative, $\dot{P}\left(10^{-19} \mathrm{~s} \mathrm{~s}^{-1}\right)$ & $9.01(5)$ \\
Dispersion measure, $\mathrm{DM}\left(\mathrm{pc} \mathrm{cm}^{-3}\right)$ & $476(5)$ \\
Projected semi-major axis ${ }^{a}, a$ sin $i(\mathrm{~s})$ & $34.7827(5)$ \\
Eccentricity, $e$ & $0.828011(9)$ \\
Epoch of periastron, $T_{0}(\mathrm{MJD})$ & $50875.02452(3)$ \\
Orbital period, $P_{B}(\mathrm{~d})$ & $18.7791691(4)$ \\
Longitude of periastron, $\omega(\mathrm{deg})$ & $127.6577(11)$ \\
Advance of periastron, $\dot{\omega}(\mathrm{deg}$ yr & $-1)$ \\
Flux density at $3100 \mathrm{MHz}, S_{3100}(\mathrm{mJy})$ & $0.0090(2)$ \\
& $0.34(7)$ \\
Time span $(\mathrm{MJD})$ & $50842-53624$ \\
N. of ToAs & 635 \\
RMS $(\mu s)$ & 851.173 \\
\hline Derived parameters ${ }^{b}$ & \\
Characteristic age, $\tau_{\mathrm{c}}\left(10^{9} y r\right)$ & 1.83 \\
Surface magnetic field, $B_{0}\left(10^{9} \mathrm{G}\right)$ & 9.80 \\
Total mass $M_{\mathrm{TOT}}\left(M_{\odot}\right)$ & $2.57(10)$ \\
Mass function $f\left(M_{\mathrm{C}}\right)\left(M_{\odot}\right)$ & $0.128121(5)$ \\
Orbital separation $A(1 \mathrm{~s})$ & $94.4(6)$ \\
Minimum companion mass $M_{\mathrm{C}, \mathrm{min}}\left(M_{\odot}\right)$ & 0.93 \\
\hline
\end{tabular}

${ }^{a}$ The projected semi-major axis $a \sin i$ is the semi-major axis of the projection of the orbit of the pulsar, around the system's center of mass, onto the plane containing the line of sight and the line of nodes.

${ }^{b}$ Characteristic age and surface magnetic field have been calculated using standard formulas, namely $\tau_{\mathrm{c}}=P / 2 \dot{P}$ and $B_{0}=3.2 \times$ $10^{19} \sqrt{P \dot{P}} \mathrm{G}$. The total mass $M_{\mathrm{TOT}}$ has been calculated from the relativistic periastron advance and the measured Keplerian parameters, assuming the validity of general relativity. The minimum companion mass was estimated using the observed mass function $f\left(M_{\mathrm{C}}\right)$ and the lower limit for the total mass, as given by its uncertainty, in the case of $\sin i=1$. For details see Lorimer \& Kramer (2005).

The derived values for the characteristic age $\left(\tau_{\mathrm{c}}=1.83 \times\right.$ $\left.10^{9} \mathrm{yr}\right)$ and the surface magnetic field $\left(B=9.8 \times 10^{9} \mathrm{G}\right)$, as well as the combined values of the spin period $(P=104 \mathrm{~ms})$ and its period derivative $\left(\dot{P}=9 \times 10^{-19}\right)$, indicate that PSR J1811-1736 is a neutron star that experienced a spin-up phase via accretion from mass overflowing from its companion. These parameters, in conjunction with the measured orbital eccentricity ( $e=0.828$ ), indeed suggest that PSR J1811-1736 is a mildly recycled pulsar whose companion star was massive enough to also undergo a SN explosion. This second SN imparted the actually observed large eccentricity to the system (e.g. Bhattacharya \& van den Heuvel 1991).

Our new measurement of the relativistic periastron advance, $\dot{\omega}=0.0090 \pm 0.0002 \mathrm{deg} \mathrm{yr}^{-1}$, allows us to determine the value of $2.57 \pm 0.10 M_{\odot}$ for the total mass of the system, assuming that general relativity is the correct theory of gravity and that the observed value is fully due to relativistic effects (e.g. Damour \& Deruelle 1986). This value, combined with the measured mass function, implies a minimum companion mass of $0.93 M_{\odot}$.

The value for the total mass is relatively low, but very similar to the total mass of the double pulsar system (Lyne et al. 2004) and the recently discovered DNS system PSR J1756-2251 (Faulkner et al. 2005). In fact, these systems have neutron star 
companions that have the lowest neutron star masses observed so far, $M_{\mathrm{c}}=1.25 M_{\odot}$ and $M_{\mathrm{c}}=1.17 M_{\odot}$ (for a recent review see Stairs 2004), respectively. In Fig. 2 we show the so-called mass-mass diagram where the pulsar and companion masses can be directly compared. The measured value for the advance of periastron means that the sum of the masses must lie along the diagonal line, while the constraint on the inclination $\sin i \leq 1$ excludes the hatched region below the dotted line. Assuming that the neutron stars in this system must have a mass which is larger than the lowest mass so-far measured, i.e. $1.17 M_{\odot}$, we find that they both have masses in the interval $1.17 M_{\odot} \leq M_{P}, M_{C} \leq 1.50 M_{\odot}$. This interval contains all but the heaviest neutron stars masses for which a reliable determination has been obtained. Using this mass constraint, we can also translate this range into lower and upper limits on the inclination of the system, i.e. $44 \mathrm{deg} \lesssim i \lesssim 50 \mathrm{deg}$.

Alternatively, if either the pulsar or the companion have a mass equal to the observed median neutron star mass of $1.35 M_{\odot}$ (Stairs 2004), the other neutron star would have a mass of $1.22 \pm 0.10 M_{\odot}$. This value is consistent with the lower limit in the previous discussion, but this also allows for the possibility that one of the two neutron stars has a mass as low as $1.12 M_{\odot}$.

\section{Future potential of timing observations}

In order to determine the companion mass without ambiguities, it is necessary to measure a second post-Keplerian (PK) parameter (e.g. Damour \& Taylor 1992). We have investigated the possibility of measuring the PK parameter $\gamma$, which describes the combined effect of gravitational redshift and a second order Doppler effect. For a companion mass of $1.35 M_{\odot}$, the expected value is $\gamma=0.021 \mathrm{~ms}$. Using simulated data sets for the presently available timing precision, we estimate that a $3 \sigma$ detection for $\gamma$ is achievable after about 4 more years of observation. However, in order to obtain a $10 \%$ accuracy in mass measurement by determining $\gamma$ to a similar precision, several decades of observations may be needed.

From similar simulations, we estimate that PK parameters like the rate of orbital decay, $\dot{P}_{\mathrm{B}}$, or the Shapiro delay, are unmeasurable in this system, unless significant improvements in timing precision can be obtained. For a companion mass of $1.35 M_{\odot}, \dot{P}_{\mathrm{B}}$ is only $-9.4 \times 10^{-15} \mathrm{~s} \mathrm{~s}^{-1}$ and we expect an amplitude of only $6 \mu$ s for the amplitude of the Shapiro delay. We also note that the effects of geodetic precession (see e.g. Kramer 1998) will not be measurable within a reasonable time, as it has a period of order of $10^{5}$ years.

\section{Improving timing precision}

It is obvious that the measurement of further PK parameters will only be possible if higher timing precision can be achieved for this pulsar. For instance, if a precision of $50 \mu$ s could be obtained, $\gamma$ could be measurable to a $10 \%$ accuracy after a total of just $5 \mathrm{yr}$ of observations, while a $3 \sigma$ detection of the orbital decay may be achieved after about $6 \mathrm{yr}$.

One way to achieve higher timing precision is to detect narrow features in the observed pulse profile by means of higher effective time resolution. This is most commonly achieved through better correction for dispersion smearing that is caused by the radio signal's passage through the ionized interstellar medium. While this effect is actually completely removed by the use coherent de-dispersion techniques (see Hankins \& Rickett 1975) at some of our telescopes, it is apparent that the current timing

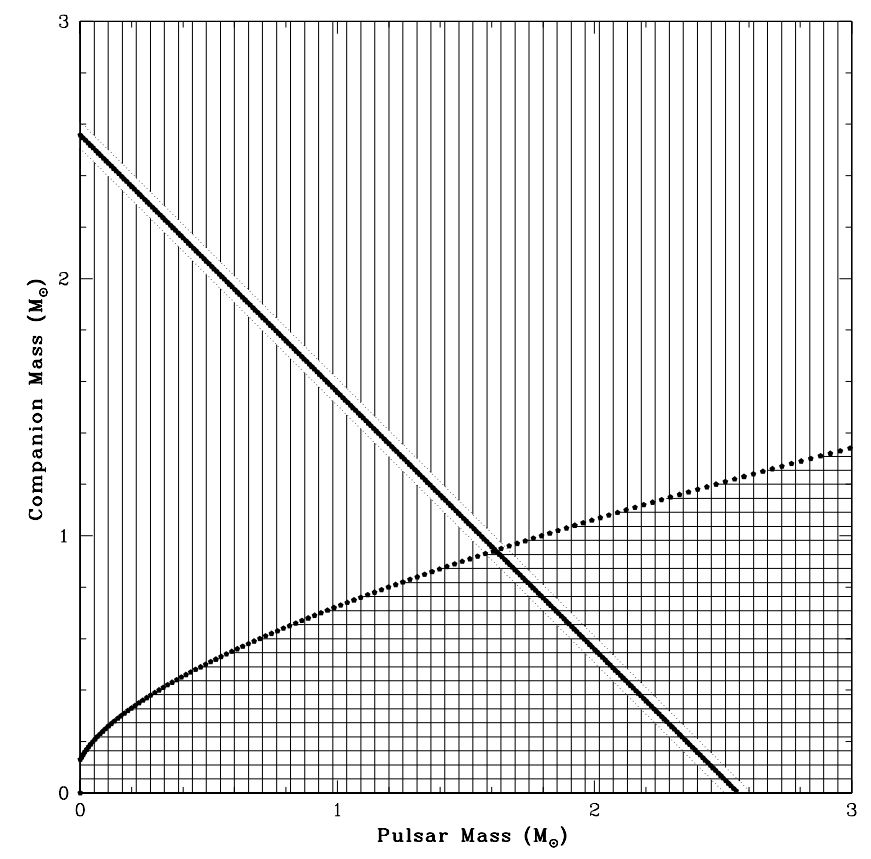

Fig. 2. Mass-mass diagram for the binary system hosting PSR J18111736. The shaded area below the dotted curved line is excluded because of the geometrical constraint $\sin i \leq 1$, while the area outside the diagonal stripe is excluded by the measurement of the relativistic periastron advance and the derived value for the total mass for this system.

precision is limited instead by broadening of the pulse profile due to interstellar scattering (Löhmer et al. 2004, and references therein). Indeed, the pulse profile at $1.4 \mathrm{GHz}$ shows a strong scattering tail (see Fig. 3) which prevents a highly accurate determination of the pulse time of arrival. As scattering is a strong function of observing frequency, we can expect to reduce its effect, and hence to enable higher timing precision, by using timing observations at frequencies above $1.4 \mathrm{GHz}$.

We obtained observations at $3.1 \mathrm{GHz}$ that confirm this expectation. The pulse profile obtained at this frequency shows no evidence of interstellar scattering, and its width at $10 \%$ is only $7.3 \mathrm{~ms}$. This is a great improvement with respect to the $1.4 \mathrm{GHz}$ profile, whose $10 \%$ width is $58.3 \mathrm{~ms}$. A flux density of $0.34 \pm 0.07 \mathrm{mJy}$ measured at $3.1 \mathrm{GHz}$ suggests that regular timing observations at this frequency should be possible and should significantly improve the achievable timing precision. This would allow us to measure a second PK parameter to an accuracy that is sufficiently precise to determine the companion mass.

Using the data available at $1.4 \mathrm{GHz}$ and $3.1 \mathrm{GHz}$, we computed spectral indexes for flux density and scattering time. For the flux density we obtain a spectral index of $\beta=-1.8 \pm 0.6$. Subdividing our observing band at $1.4 \mathrm{GHz}$ we obtain two different profiles that we use to measure the pulse scatter timescale $\tau$ by applying the technique described in Löhmer et al. 2001. We convolve the $3.1 \mathrm{GHz}$-profile, assumed to represent the true pulse shape, with an exponential scattering tail and obtain scattering times by a least-square comparison of the convolved profile with the observed pulse shape. At $1.284 \mathrm{GHz}$ we find $\tau_{\mathrm{s}}=16.9 \mathrm{~ms}$, and $\tau_{\mathrm{s}}=10.6 \mathrm{~ms}$ at $1.464 \mathrm{GHz}$, respectively. This results in a spectral index $\alpha$ of the scattering time, i.e. $\tau \propto v^{-\alpha}$, of $\alpha=3.5 \pm 0.1$. Such a measured value agrees very well with analogous results from Löhmer et al. (2001; 2004) who determined $\alpha$ for a number of pulsars with very high dispersion measures. 

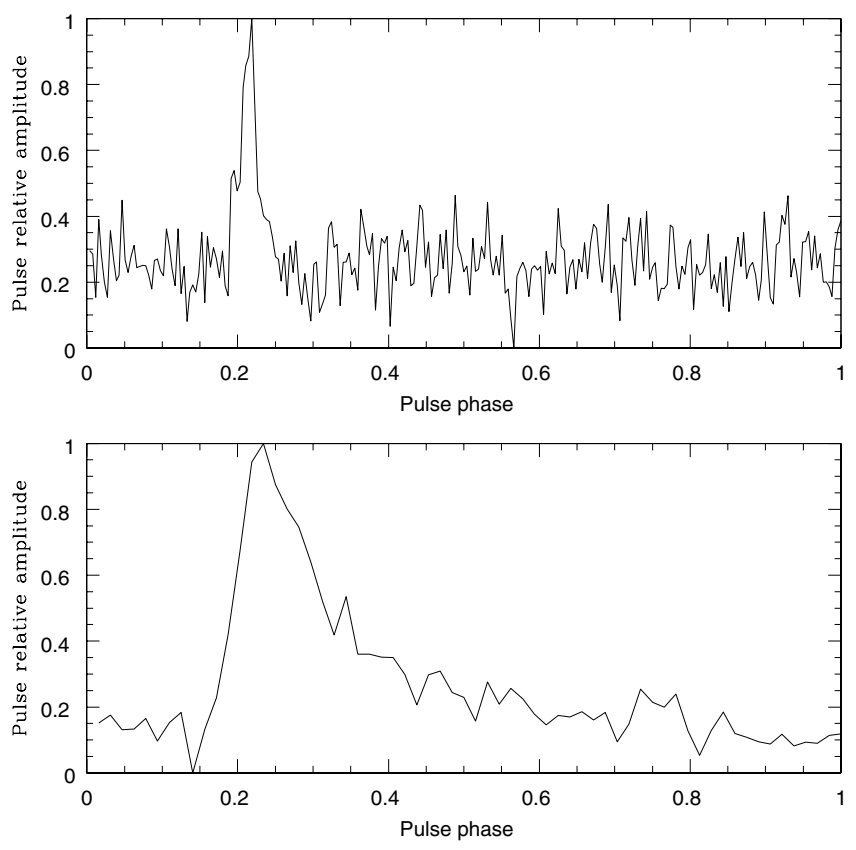

Fig. 3. Pulses' profiles of PSR J1811-1736 at $1.4 \mathrm{GHz}$ (bottom panel) and $3.1 \mathrm{GHz}$ (top panel). Both profiles have been obtained with $10 \mathrm{~min}$ observations performed with the Parkes radio telescope in February 2005.

The measured spectral index of the scattering time is also consistent with the fact that the pulsar has not been detected at frequencies below $1 \mathrm{GHz}$. For example at $400 \mathrm{MHz}$ we calculate $\tau_{\mathrm{s}} \sim 1 \mathrm{~s}$ which is almost an order of magnitude greater than the spin period of the pulsar thus making it impossible to detect it as a pulsating source.

\section{Previous searches for pulsations from the companion}

Searches for pulsations from the binary companion of PSR J1811-1736 have been performed on Parkes and Effelsberg data. Parkes observations have been investigated with the procedure described in Faulkner et al. (2004), while Effelsberg data have been processed using the procedure described in Klein (2004). Both searches were unsuccessful in detecting any evidence of pulsation. The very high value of the dispersion measure for this system may suggest that the interstellar scattering is responsible for the failure in detecting any pulsation. Therefore we studied the possible impact of this phenomenon on our searches for pulsations from the companion of PSR J1811-1736.

We considered $1 \mathrm{hr}$ observations done with the Effelsberg telescope using either the $20 \mathrm{~cm}(1.4 \mathrm{GHz})$ or the $11 \mathrm{~cm}$ $(2.7 \mathrm{GHz})$ receiver, exploring a range of possible flux densities ( $S=0.05,0.5,1 \mathrm{mJy})$ and a detection in a signal-to-noise ratio threshold of $S / N=10$. Using the DM of the observed pulsar, and assuming Effelsberg observations at $1.4 \mathrm{GHz}$, we find a minimum detectable period of $P_{\min }=750 \mathrm{~ms}$ for a flux density of $S=50 \mu \mathrm{Jy}$, while even for the flux densities of $S=500 \mu \mathrm{Jy}$ and $S=1$ mJy periods below $\sim 10 \mathrm{~ms}$ become undetectable at the observing frequency of $1.4 \mathrm{GHz}$.

At $2.7 \mathrm{GHz}$, the system performance of the Effelsberg telescope allows for an antenna gain of $G=1.5 \mathrm{~K} \mathrm{Jy}^{-1}$ with a system temperature $T_{\text {sys }}=17 \mathrm{~K}$. Using these parameters, we obtain minimum periods of $P_{\min }=110 \mathrm{~ms}, P_{\min }=2.5 \mathrm{~ms}$ and
$P_{\min }=1.6 \mathrm{~ms}$ for flux densities $S_{2.7 \mathrm{GHz}}=50 \mu \mathrm{Jy}, 500 \mu \mathrm{Jy}$ and 1 mJy respectively.

When searching for pulsations from the binary companion of a Galactic recycled pulsar in a double neutron star system, it is more likely that the companion is a young pulsar with rather ordinary spin parameters, as found for PSR J0737-3039B in the double pulsar system (Lyne et al. 2004). Our lower limits on the minimum detectable period therefore suggests that interstellar scattering should not have prevented the detection of the companion, unless it were a very fast spinning or very faint source.

\section{Constraints on the kick velocity of the second $\mathrm{SN}$ explosion}

The large eccentricity of J1811-1736 system can be ascribed to a sudden loss of mass which results in a change of the orbital parameters. Such a sudden loss of mass can be attributed to the SN explosion that formed the younger unseen neutron star companion (see, e.g., Bhattacharya \& van den Heuvel 1991). Under the hypothesis of a symmetric explosion, simple calculations show that the binary survives this event only if the expelled mass $M_{\exp }$ is less than half of the total mass of the binary before the explosion (pre-SN binary). The induced eccentricity is a simple function of the amount of the expelled mass: $e=M_{\exp } / M_{\mathrm{TOT}}$, where $M_{\mathrm{TOT}}$ is the total mass of the pre-SN binary. In the case of the binary system hosting PSR J1811-1736, the measured eccentricity, $e=0.828$, and the derived total mass, $M_{\text {bin }}=2.57 M_{\odot}$, would imply a total mass $M_{\mathrm{TOT}}=4.7 M_{\odot}$ for the pre-SN binary.

The high space velocities measured for isolated pulsars indicate that neutron stars may receive a kick when formed, with an unpredictable amplitude and direction (Hansen \& Phinney 1997; Cordes \& Chernoff 1998; Arzoumanian et al. 2002). Such kicks imparted to the newly formed neutron stars are caused by asymmetric supernova explosions. If an asymmetric SN explosion occurs in a binary system, the survival and the eventual post-SN binary parameters are jointly determined by the mass loss and the vector representing the velocity imparted to the neutron star. In this case a simple survival condition like the one derived for the symmetric explosion case cannot be determined.

A correlation between the pulsar's spin period and orbital eccentricity has recently been found for DNS systems (McLaughlin et al. 2005, Faulkner et al. 2005). A numerical simulation by Dewi et al. (2005) linked this correlation to the typical amplitude of the kick velocity received by the younger neutron star at birth. Dewi et al. (2005) found that the spin period versus eccentricity correlation is recovered if the typical kick amplitude satisfies the condition $V_{\mathrm{K}} \lesssim 50 \mathrm{~km} \mathrm{~s}^{-1}$.

To investigate the nature of the kick received by the younger neutron star in this system we considered as the pre-SN binary a binary system containing a neutron star and a helium star. We then constrained the total mass of this system by combining our results on the total mass of the actual binary with the range given by Dewi \& Pols (2003) for the mass of the helium star that was the companion of PSR J1811-1736 before the explosion. The helium star mass range $2.8 M_{\odot} \leq M_{\mathrm{C}} \leq 5.0 M_{\odot}$ given by Dewi \& Pols (2003) leads to a total mass range of $4.0 M_{\odot} \leq M_{\mathrm{TOT}} \leq 6.5 M_{\odot}$.

Binary parameters for the pre-SN binary have been chosen as follows. The eccentricity has been assumed negligible, since the accretion phase responsible for spinning up the pulsar also provided strong tidal forces that circularised the orbit. The orbital separation has been constrained to be between the the minimum (pericentric) and maximum (apocentric) distance between 
the two neutron stars in the post-SN binary. This statement can be justified as follows. The typical velocity of the expelled matter in a SN explosion is close to the speed of light, while the typical orbital velocity of the stars in a binary system is of order of $\sim 100 \mathrm{~km} \mathrm{~s}^{-1}$, using for the total mass any value in the range we used for $M_{\text {TOT }}$ and a value for the orbital separation comparable to the one for the present binary system, i.e. few light-minutes (see the discussion in the next paragraph of the post-SN binary evolution due to general relativistic effects). This means that the change in position of the two stars is negligible if compared to the change in position of the expelled matter. The time required for the binary system to do the transition from the pre-SN to the post-SN binary is the time required by the expelled matter to travel along a path as long as the orbital separation, i.e. few minutes. After such an elapsed time the matter expelled in the SN explosion encloses both stars and has no more gravitational effects on their binary motion. This time is also much shorter than the orbital period of few days for a pre-SN binary like the one we are considering. This means that during this transition the positions of the two stars remained unchanged, and their distance was a distance periodically assumed by the two stars also in their orbital motion in the post-SN binary.

To make a fully consistent comparison between the actually observed binary and the eccentric binary that emerged from the last SN explosion (post-SN binary) one has to take into account the secular changes of the orbital parameters caused by general relativistic effects. In order to do this one needs to have an estimate for the time since the last SN. The only timescale that is available to us is the characteristic age of the first born pulsar. We then find binary parameters that are consistent with the present system values within their uncertainties. Given the well known uncertainties in this age estimation, we considered the possibility that the present binary is in fact up to ten times older than suggested by the characteristic age (i.e $1.8 \times 10^{10} \mathrm{yr}$ ). Even when considering this extreme age, we find that our results remain unaffected. We consequently decided to use as post-SN orbital parameters the same values we measure today.

By insisting that the total energy and total angular momentum, calculated in the center-of-mass frame, of the post-SN binary and the present systems are conserved, we can combine these terms to obtain an equation for the kick amplitude, as a function of the two angles, representing its direction in a suitable reference frame, and the total mass and orbital separation before the explosion. We assumed that the probability of occurrence for any given kick vector is proportional to the solid angle described by the direction of the kick in spherical coordinates and then calculated the probability of having a kick velocity lower than some fixed values. We chose the values of 50,100 and $150 \mathrm{~km} \mathrm{~s}^{-1}$ (hereafter $P_{50}, P_{100}$ and $P_{150}$ respectively.). Figure 4 shows that $P_{50}$ is not negligible if the total mass of the pre-SN binary system is lower than $6 M_{\odot}$. Moreover, all considered probabilities peak in correspondence of a total pre-SN mass of $4.70 M_{\odot}$, corresponding to the null kick case. These results lead to the conclusion that the younger neutron star in this system received a low velocity kick and is thus similar to all other known DNS systems, which all have tighter orbits.

Nevertheless, the binary system containing PSR J1811-1736 is much wider than all other known DNS systems. This may indicate that the binary evolution of this system may have been (at least partially) different. In particular the wide orbital separation for this system may be compatible with an evolution during which the pulsar's progenitor avoided completely a common envelope phase (Dewi \& Pols 2003) or that this phase was too short to sufficiently reduce the orbital separation. Moreover if

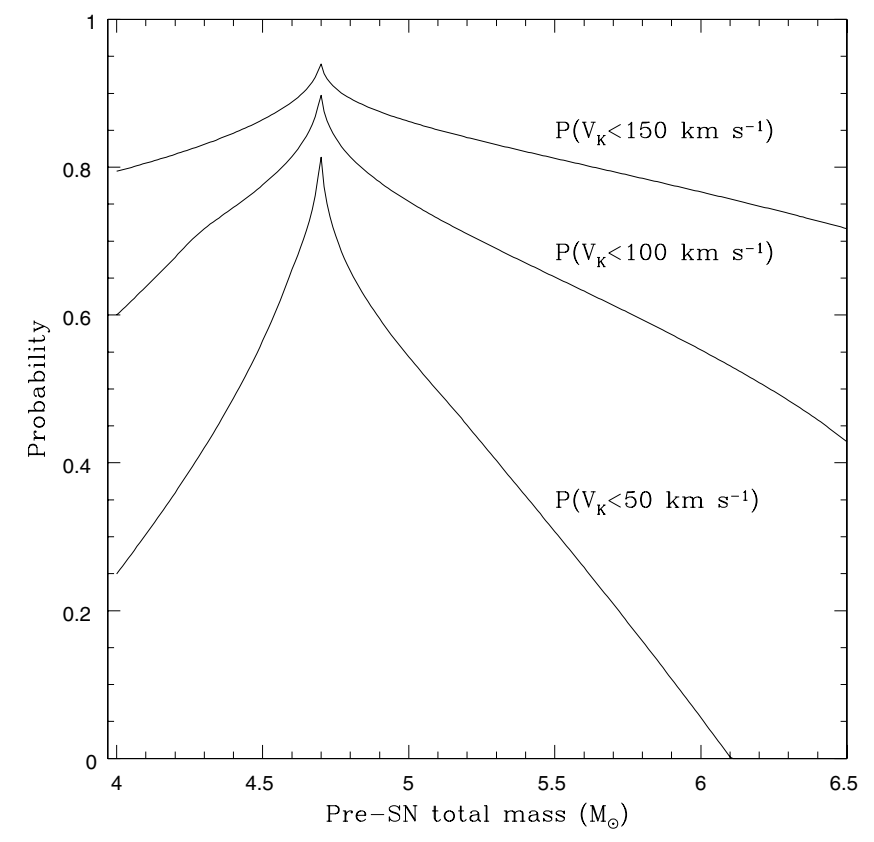

Fig. 4. Probabilities to have a kick velocity lower than or equal to 50 (lower line), 100 and 150 (upper line) $\mathrm{km} \mathrm{s}^{-1}$ as a function of the pre$\mathrm{SN}$ total mass. All probabilities peak in correspondence of a pre-SN total mass $M_{\text {TOT }}=4.70 M_{\odot}$, which is the mass of the binary system before the explosion in the case of a symmetric SN. The probability to have a kick velocity lower than $50 \mathrm{~km} \mathrm{~s}^{-1}$ is not negligible for all but the highest considered values for the binary pre-SN mass.

the spin-up occurred via the stellar wind of the giant companion, then the system would tend to be wider due to the isotropic mass loss from the companion (Dewi et al. 2005).

\section{Summary and conclusions}

We have presented an improved timing solution for the binary pulsar J1811-1736. This solution improves the previously measured values for the spin and Keplerian orbital parameters and one post-Keplerian orbital parameter, the periastron advance. These results would not have been achieved without data from the three telescopes used and are the first obtained as part of the European Pulsar Timing Array (EPTA) collaboration.

The measured values for the spin period and its first derivative are typical of a mildly recycled neutron star, while the high eccentricity of the binary system can be seen as a signature of the $\mathrm{SN}$ explosion that interrupted the mass transfer from the companion to the accreting neutron star. This is likely to have occurred before the pulsar could reach spin periods typical of the fully recycled (i.e. millisecond) pulsars. This leads to the conclusion that PSR J1811-1736 is a member of a DNS binary system.

The determined value of the periastron advance provides further confirmation for this scenario as it suggests a total mass of the system of $M_{\mathrm{tot}}=2.57 \pm 0.10 M_{\odot}$. This value is similar to the total mass of two other DNS systems, i.e. the double pulsar (Lyne et al. 2004) and PSR J1756-2251 (Faulkner et al. 2005). In both these systems, the non-recycled neutron stars is very light. Assuming that PSR J1811-1736 is a neutron star with a mass within the currently measured mass range for neutron stars, we find the companion mass to lie in the same range. Using these arguments we determine the inclination of the orbital plane to within $\sim 6$ degrees. 
We also investigated the possibility of measuring a second post-Keplerian parameter, in order to determine both masses and thus to definitively determine the nature of the companion. Unfortunately, the pulse profile at $1.4 \mathrm{GHz}$ is heavily broadened by interstellar scattering which limits the timing precision and means that a second post-Keplerian parameter is not measurable within a reasonable amount of time with observations at that frequency. However we find that at $3 \mathrm{GHz}$ the scattering is sufficiently reduced and the flux density is sufficiently high that higher precision timing will be possible at this frequency. Comparing the pulse profiles at 1.4 and $3 \mathrm{GHz}$ we find that the scattering timescale for this pulsar scales with frequency with a power law of index $\alpha=3.5 \pm 0.1$ which is in excellent agreement with earlier results on high dispersion measure pulsars.

Considering the effects of the interstellar scattering on the detectability of pulsations from the companion, we find that the minimum detectable period is longer at lower frequencies and for fainter objects. In general, we do not expect interstellar scattering to be the cause for the continued non-detection of the companion neutron star.

The orbital separation for this system is much wider than for all other DNS systems, and it suggests that its binary evolution has been different. One explanation invokes the lack of a common envelope phase, during which the size of the orbit shrinks due to tidal forces in the envelope of the companion star. Another explanation (Dewi et al. 2005), not necessarily conflicting with the previous one, invokes a different mass transfer mechanism in the spin-up phase of the pulsar, namely via stellar wind, while all other recycled pulsars in the known DNS have been spun up via Roche lobe overflow mass transfer.

Finally, we investigated the kick imparted to the second born neutron star during the second SN. We find that for realistic values of the total mass of the pre-SN binary, the kick velocity has a not negligible probability of being lower than $50 \mathrm{~km} \mathrm{~s}^{-1}$. This constraint is common to all DNS systems, as shown by Dewi et al. (2005). This evidence for a low amplitude asymmetric kick received by the younger neutron star may be the consequence of the effects of binary evolution on a star that undergoes a SN explosion, effects that somehow are able to tune the amplitude of such kick.

Note added in proof. Our results on the natal kick received by the companion of PSR J1811-1736 support a low-amplitude kick scenario for the neutron star companion of a mildly recyled binary pulsar. However, previous and recent investigations on other double neutron star systems by other authors lead to different conclusions, namely they find that high-amplitude kicks are more likely to have occurred in the supernova that formed the pulsar's companion. We believe that therefore a variety of kick amplitudes is possible. More studies are needed to establish which conditions need to be met for high or low amplitude kicks. In order to give an insight into a more complete picture, we refer the interested reader to, e.g., Fryer \& Kalogera (1997), Willems et al. (2006) and Stairs et al. (2006).

\section{References}

Arzoumanian, Z., Chernoff, D. F., \& Cordes, J. M. 2002, ApJ, 568, 289 Backer, D. C., Dexter, M. R., Zepka, A., et al. 1997, PASP, 109, 61 Bhattacharya, D., \& van den Heuvel, E. P. J. 1991, Phys. Rep., 203, 1 Cordes, J. M., \& Chernoff, D. F. 1998, ApJ, 505, 315

Damour, T., \& Deruelle, N. 1986, Ann. Inst. H. Poincaré (Physique Théorique), 44, 263

Damour, T., \& Taylor, J. H. 1992, Phys. Rev. D, 45, 1840

Dewi, J. D. M., Podsiadlowski, P., \& Pols, O. R. 2005, MNRAS, 363, L71

Dewi, J. D. M., \& Pols, O. R. 2003, MNRAS, 344, 629

Downs, G. S., \& Reichley, P. E. 1983, ApJS, 53, 169

Faulkner, A. J., Stairs, I. H., Kramer, M., et al. 2004, MNRAS, 355, 147

Faulkner, A. J., Kramer, M., Lyne, A. G., et al. 2005, ApJ, 618, L119

Fryer, C. \& Kalogera, V. 1997, ApJ, 489, 244

Hankins, T. H., \& Rickett, B. J. 1975, in Methods in Computational Physics Vol. 14 - Radio Astronomy (New York: Academic Press), 55

Hansen, B., \& Phinney, E. S. 1997, MNRAS, 291, 569

Hobbs, G., Lyne, A. G., Kramer, M., Martin, C. E., \& Jordan, C. A. 2004, MNRAS, 353, 1311

Klein, B. 2004, Ph.D. Thesis, University of Bonn

Kramer, M. 1998, ApJ, 509, 856

Kramer, M., Xilouris, K. M., Lorimer, D. R., et al. 1998, ApJ, 501, 270

Kramer, M., Lange, C., Lorimer, D. R., et al. 1999, ApJ, 526, 957

Lange, C. 1999, Ph.D. Thesis, University of Bonn

Löhmer, O., Kramer, M., Mitra, D., Lorimer, D. R., \& Lyne, A. G. 2001, ApJ, 562, L157

Löhmer, O., Mitra, D., Gupta, Y., Kramer, M., \& Ahuja, A. 2004, A\&A, 425, 569

Lorimer, D. R., \& Kramer, M. 2005, Handbook of Pulsar Astronomy (Cambridge University Press)

Lyne, A. G., Burgay, M., Kramer, M., et al. 2004, Science, 303, 1153

Lyne, A. G., Camilo, F., Manchester, R. N., et al. 2000, MNRAS, 312, 698

Manchester, R. N., Lyne, A. G., Camilo, F., et al. 2001, MNRAS, 328, 17

McLaughlin, M., Lorimer, D., Champion, D., et al. 2005, in Binary Radio Pulsars, ASP Conf. Ser. 328: 43

Mignani, R. P. 2000, A\&A, 358, L53

Stairs, I. H. 2004, Science, 304, 547

Stairs, I. H., Thorsett, S. E., Dewey, R. J., Kramer, M., \& McPhee, C. A. 2006, MNRAS, 373, L50

Standish, E. M. 1990, A\&A, 233, 252

Taylor, J. H. 1992, Philos. Trans. Roy. Soc. London A, 341, 117

Taylor, J. H., \& Weisberg, J. M. 1989, ApJ, 345, 434

Voûte, J. L. L., Kouwenhoven, M. L. A., van Haren, P. C., et al. 2002, A\&A, 385, 733

Willems, B., Kaplan, J., Fragos, T., Kalogera, V. \& Belczynski, K. 2006, Phys. Rev. D, 74, 043003 